\title{
Exact Mass-Coupling Relation for the Homogeneous Sine-Gordon Model
}

\author{
Zoltán Bajnok, ${ }^{1, *}$ János Balog, ${ }^{1, \dagger}$ Katsushi Ito, ${ }^{2, \$}$ Yuji Satoh, ${ }^{3,8}$ and Gábor Zsolt Tóth ${ }^{1, \|}$ \\ ${ }^{1}$ MTA Lendület Holographic QFT Group, Wigner Research Centre, H-1525 Budapest 114, P.O.B. 49, Hungary \\ ${ }^{2}$ Department of Physics, Tokyo Institute of Technology, Tokyo 152-8551, Japan \\ ${ }^{3}$ Institute of Physics, University of Tsukuba, Tsukuba, Ibaraki 305-8571, Japan
}

(Received 10 December 2015; revised manuscript received 15 March 2016; published 2 May 2016)

\begin{abstract}
We derive the exact mass-coupling relation of the simplest multiscale quantum integrable model, i.e., the homogeneous sine-Gordon model with two mass scales. The relation is obtained by comparing the perturbed conformal field theory description of the model valid at short distances to the large distance bootstrap description based on the model's integrability. In particular, we find a differential equation for the relation by constructing conserved tensor currents, which satisfy a generalization of the $\Theta$ sum rule Ward identity. The mass-coupling relation is written in terms of hypergeometric functions.
\end{abstract}

DOI: 10.1103/PhysRevLett.116.181601

Introduction.-One of the most difficult problems in a quantum field theory is to determine the mass-coupling relation, i.e., the relation between the renormalized couplings related to the Lagrangian definition of the theory and the physical masses. Such an exact relation would express, for example, the dynamically generated nucleon mass in the chiral limit of quantum chromodynamics in units of the perturbative Lambda-parameter $\Lambda$ which is defined in, say, the $\overline{\mathrm{MS}}$ scheme. The difficulty lies in the fact that the Lagrangian is defined at short distances (or ultravioletUV_-scale), while the masses are the parameters at large distances (or infrared-IR-scale).

There is one family of models where such a relation can be found exactly, namely, two dimensional integrable models. The mass $/ \Lambda$ ratio was indeed exactly determined $[1,2]$ in the nonlinear sigma (NLS) model. To this end, one adds an external field coupled to one of the conserved charges, calculates the free energy perturbatively on the UV side, and compares it to the large field expansion from the Bethe Ansatz integral equation or the thermodynamic Bethe Ansatz (TBA) equation [3] on the IR side. Later this method was applied to many other models [4-10].

In contrast to the NLS model with marginally relevant perturbations, there is also a large class of integrable models which can be defined as perturbations of their UV-limiting conformal field theories (CFTs) by strictly relevant scaling operators. In this case, coupling constants are dimensionful, and one can show $[11,12]$ that they are not renormalized in the perturbative CFT scheme and hence, are physical themselves. When a model in this class has only one perturbing operator, the relation between the coupling constant and the (lowest) physical mass boils down to a single proportionality constant. This nontrivial constant was determined as well by the method described above for the sine-Gordon and affineToda field theories and their reductions $[13,14]$.

A common feature of all these models is that they have only one mass scale. In some of these models, the particles have a nontrivial spectrum but all mass ratios are encoded in the $S$ matrix: the UV/IR relation is complete once the lowest mass is expressed by $\Lambda$, the coupling, or some other physical dimensionful parameter related to the Lagrangian. However, when the models have several independent perturbing operators, the particle spectrum continuously depends on the couplings and not fixed by the $S$ matrix. In this sense, such models can be called multiscale, to which the method in the single-scale case is not applicable, and hence, there are no results for multiscale mass-coupling relations in the literature.

The aim of this Letter is therefore to provide a novel method which can fill this gap. Though our method is conceptually more general, we focus on a class of multiscale quantum integrable models with strictly relevant perturbations, i.e., the homogenous sine-Gordon (HSG) model [15-20]. We present our ideas in particular for its simplest case with two scales. The mass-coupling relation gives the one-point functions of the perturbing operators, encoding all the nonperturbative information which is not captured by the CFT perturbation. Via the gauge-string duality, it is applied to the four-dimensional maximally supersymmetric gauge theory at strong coupling, which is one of the recent main subjects in field and string theories: it provides the missing link to derive an analytic expansion [21-24] of the strong-coupling amplitudes [25]. These are also our main motivations. Below, we analyze the model both from the UV and IR side, and compare the results to obtain the mass-coupling relation.

$U V$ : perturbed CFT.-The simplest multiscale HSG model is the perturbation of the $s u(3)_{2} / u(1)^{2}$ coset CFT by its weight- 0 adjoint primary fields. Fortunately, the coset allows an equivalent representation in terms of the projected product [26] of the Ising and the tricritical Ising (TCI) minimal models, providing a handy calculational basis: $s u(3)_{2} / u(1)^{2} \sim \mathcal{M}_{3,4} \otimes \mathcal{M}_{4,5}$, where $\mathcal{M}_{p, q}$ stands for the minimal model with central charge $c=1-6(p-q)^{2} / p q$. 
The coset chiral algebra is larger than the Virasoro algebra; thus, its diagonal modular invariant partition function representing the spectrum decomposes into the product of Virasoro characters nondiagonally as $Z=2 \chi_{\frac{1}{16} \frac{3}{80}} \overline{\frac{1}{16}}_{\frac{3}{80}}+$ $2 \chi_{\frac{1}{16} \frac{7}{16}} \bar{\chi}_{\frac{1}{16} \frac{7}{16}}+\left(\chi_{00}+\chi_{\frac{1}{2} \frac{3}{2}}\right)\left(\bar{\chi}_{00}+\bar{\chi}_{\frac{1}{2} \frac{3}{2}}\right)+\left(\chi_{\frac{1}{2} 0}+\chi_{0 \frac{3}{2}}\right)\left(\bar{\chi}_{\frac{1}{2} 0}+\bar{\chi}_{0 \frac{3}{2}}\right)+$ $\left(\chi_{0 \frac{1}{10}}+\chi_{\frac{1}{2} \frac{3}{5}}\right)\left(\bar{\chi}_{0 \frac{1}{10}}+\bar{\chi}_{\frac{1}{2} \frac{3}{5}}\right)+\left(\chi_{0 \frac{3}{5}}+\chi_{\frac{1}{2} \frac{1}{10}}\right)\left(\bar{\chi}_{0 \frac{3}{5}}+\bar{\chi}_{\frac{1}{2} \frac{1}{10}}\right), \quad$ where $\chi_{h h^{\prime}}=\chi_{h}^{(1)} \chi_{h^{\prime}}^{(2)}$ refers to the characters in the tensor product with $h, h^{\prime}$ being the dimension of primaries. The chiral algebra can be taken to be the product of the free fermion algebra generated by $\psi(z)$ of dimension $1 / 2$ on the Ising side and the superconformal algebra generated by $L^{(2)}(z), G(z)$ on the TCI part. The full Virasoro field is the $\operatorname{sum} L(z)=L^{(1)}(z)+L^{(2)}(z)$, where the Ising contribution is $L^{(1)}(z)=-(1 / 2) \psi(z) \partial \psi(z)$. There are four fields of dimension $(3 / 5,3 / 5)$, which can be obtained from $\Phi(z, \bar{z}) \equiv$ $\Phi_{1 / 10,1 / 10}(z, \bar{z})$ by acting with the left and right chiral generators:

$$
\Phi_{i j}(z, \bar{z})=\psi_{-1 / 2}^{(i)} \bar{\psi}_{-1 / 2}^{(j)} \Phi(z, \bar{z}),
$$

where, to streamline the notations, we introduced $\psi_{-1 / 2}^{(1)}=$ $\psi_{-1 / 2}$ and $\psi_{-1 / 2}^{(2)}=\sqrt{5} G_{-1 / 2}$. This ensures the proper normalization of the operators $\left\langle\Phi_{i j} \mid \Phi_{k l}\right\rangle=\delta_{i k} \delta_{j l}$. The Lagrangian of the HSG theory is defined to be

$$
\mathcal{L}=\mathcal{L}_{\mathrm{CFT}}-\lambda_{i} \bar{\lambda}_{j} \Phi_{i j}(z, \bar{z}),
$$

where summation is understood for $i=1,2$ and $j=1,2$. Since the transformations $\lambda_{i} \rightarrow \beta \lambda_{i}$ and $\bar{\lambda}_{i} \rightarrow \beta^{-1} \bar{\lambda}_{i}$ with $\beta$ being constant do not change the perturbation, we have effectively three parameters. We also have further discrete symmetries: The remnant of the $S_{3}$ Weyl symmetry in the coset translates into the $\lambda_{i} \rightarrow \omega_{i j} \lambda_{j}$ invariance of the perturbation, where $\omega_{i j}$ stands for the rotation by $\pm 2 \pi / 3$ or the reflection $\lambda_{1} \rightarrow-\lambda_{1}$. We have similar independent transformations for the right chiral half.

IR: scattering theory.-The Hilbert space on the IR side contains the scattering states $\left|\theta_{1}, \ldots, \theta_{n}\right\rangle_{a_{1} \ldots a_{n}}$ of two types of particles with masses $m_{1}$ and $m_{2}$ which can take arbitrary values. Here, $\theta_{j}$ is the rapidity of the $j$ th particle of type $a_{j}$ whose energy is $E=m_{a_{j}} \cosh \theta_{j}$. The theory is integrable, and the two particle scattering matrix contains one resonance parameter $\sigma$ [18]:

$$
S_{12}(\theta-\sigma)=-S_{21}(\theta+\sigma)=\tanh \frac{1}{2}\left(\theta-i \frac{\pi}{2}\right) .
$$

These fermionic particles scatter on themselves trivially: $S_{11}(\theta)=S_{22}(\theta)=-1$. Our aim is to express the three IR parameters, $m_{1}, m_{2}$, and $\sigma$ in terms of the UV parameters $\lambda_{i}$ and $\bar{\lambda}_{j}$. Since the UV parameters depend on the choice of the basis for $\Phi_{i j}$, we have to map these operators to their IR counterparts. On the IR side, operators are characterized by their form factors. For a local operator $X$, they are denoted by

$$
\left\langle 0|X| \theta_{1}, \ldots, \theta_{n}\right\rangle_{a_{1} \ldots a_{n}}=F_{a_{1}, \ldots, a_{n}}^{X}\left(\left\{\theta_{i}\right\}\right) .
$$

These form factors have the structure

$$
F_{a_{1} \ldots a_{n}}^{X}\left(\left\{\theta_{i}\right\}\right)=Q_{a_{1} \ldots a_{n}}^{X}\left(\left\{x_{i}\right\}\right) \prod_{j<k} F_{a_{j} a_{k}}\left(\theta_{j}, \theta_{k}\right),
$$

where $x_{i}=e^{\theta_{i}}$, and the two particle form factors are

$$
F_{11}\left(\theta_{1}, \theta_{2}\right)=F_{22}\left(\theta_{1}, \theta_{2}\right)=-\frac{\sinh \frac{\theta_{1}-\theta_{2}}{2}}{2 \pi\left(x_{1}+x_{2}\right)}
$$

and $F_{12}\left(\theta_{1}, \theta_{2}\right) \equiv f\left(\theta_{1}-\theta_{2}\right)$, which is the minimal solution of the equation $f(\theta)=S_{12}(\theta) f(\theta+2 i \pi)$; see [27] for the details. $F_{21}\left(\theta_{1}, \theta_{2}\right)$ is then $F_{21}\left(\theta_{1}, \theta_{2}\right)=f\left(\theta_{2}-\theta_{1}\right) /$ $S_{12}\left(\theta_{2}-\theta_{1}\right)$. The factors $Q_{a_{1} \ldots a_{n}}^{X}\left(\left\{x_{i}\right\}\right)$ are polynomials in $x_{i}$ and $1 / x_{i}$. For the trace of the stress tensor, $\Theta$, they were calculated explicitly in $[27,28]$ and have the structure

$$
Q_{a_{1} \ldots a_{n}}^{\Theta}\left(\left\{x_{i}\right\}\right)=P\left(\left\{x_{i}\right\}\right)^{2} q_{a_{1} \ldots a_{n}}\left(\left\{x_{i}\right\}\right),
$$

where $P^{2}=P^{+} P^{-}$and $P^{ \pm}=P_{(1)}^{ \pm}+P_{(2)}^{ \pm}$contain the contributions of each particle type to the light cone momenta: $P_{(a)}^{ \pm}=m_{a} \sum_{j \in \text { type } a} x_{j}^{ \pm 1}$. We can easily define four local operators $X_{a b}$ by their form factors:

$$
Q_{a_{1} \ldots a_{n}}^{X_{a b}}=P_{(a)}^{+} P_{(b)}^{-} q_{a_{1} \ldots a_{n}} .
$$

We analyzed numerically the UV expansion of their two point functions by including six particles in the form factor expansion and confirmed that they all have dimensions $(3 / 5,3 / 5)$. Note that these operators depend on the masses only through the prefactors $P_{(a)}^{ \pm}$. As a consequence, their vacuum expectation values and matrix elements inherit the same mass dependence. The IR $X_{a b}$ operators are the linear combinations of the perturbing UV operators $\Phi_{i j}$, and in the following, we relate the two bases to each other.

$U V$ - IR operator relation.-In relating the UV and IR bases, note that $\Theta$ can be written in both languages,

$$
\Theta=-\frac{4}{5} \sum_{i, j} \lambda_{i} \bar{\lambda}_{j} \Phi_{i j}=\sum_{a, b} X_{a b},
$$

and its vacuum expectation value is related to the free energy density as $\mathcal{F}=-\lim _{V \rightarrow \infty}(1 / V) \ln Z=\frac{1}{2}\langle\Theta\rangle$. From the definition of the partition function, we can write

$$
\begin{array}{ll}
\partial_{i} \mathcal{F}=-\left\langle\Psi_{i}\right\rangle, & \Psi_{i}=-\bar{\lambda}_{j} \Phi_{i j}, \\
\bar{\partial}_{j} \mathcal{F}=-\left\langle\bar{\Psi}_{j}\right\rangle, & \bar{\Psi}_{j}=-\lambda_{i} \Phi_{i j},
\end{array}
$$

where $\partial_{i}$ is the shorthand for $\partial / \partial \lambda_{i}$ and similarly, $\bar{\partial}_{j}$ for $\partial / \partial \bar{\lambda}_{j}$. Form factor perturbation theory expresses the change in the particle masses in terms of the diagonal one particle form factors, $F_{a a}^{X} \equiv F_{a a}^{X}(i \pi, 0)$, of the perturbing operator as [29] 


$$
\partial_{i} m_{a}^{2}=-4 \pi F_{a a}^{\Psi_{i}}, \quad \bar{\partial}_{j} m_{a}^{2}=-4 \pi F_{a a}^{\bar{\Psi}_{j}} .
$$

The change in the scattering matrix is related to the diagonal two particle form factors $F_{a b a b}^{\Psi_{i}}(\theta) \equiv$ $\lim _{\epsilon \rightarrow 0} F_{a b a b}^{\Psi_{i}}(\theta+i \pi, i \pi, \theta+\epsilon, \epsilon)$ as [29]

$$
\begin{aligned}
8 \pi^{2} i F_{a b a b}^{\Psi_{i}}(\theta)= & 2 m_{a} m_{b} \sinh \theta \partial_{i} S_{a b}(\theta) \\
& -\left[\partial_{i} m_{a}^{2}+\partial_{i} m_{b}^{2}\right. \\
& \left.+2 \cosh \theta \partial_{i}\left(m_{a} m_{b}\right)\right] \partial_{\theta} S_{a b}(\theta) .
\end{aligned}
$$

TBA analyses relate the bulk energy density to the mass and resonance parameters as $\mathcal{F}=\frac{1}{2} m_{1} m_{2} \cosh \sigma$ (see [22]).

On the IR basis, taking into account the mass dependence of the operators $X_{a b}$, it implies for the vacuum expectation values that $\left\langle X_{a a}\right\rangle=0$ and $\left\langle X_{12}+X_{21}\right\rangle=2 \mathcal{F}$. The diagonal one particle matrix element of $\Theta$ is normalized with respect to the masses as $F_{a a}^{\Theta}(i \pi, 0)=m_{a}^{2} / 2 \pi$, which implies

$$
2 \pi F_{a a}^{X_{b c}}=\delta_{a b} \delta_{a c} m_{a}^{2} .
$$

From the explicit form of $q_{a_{1} \ldots a_{n}}$ in [27,28], one can calculate that

$$
4 \pi^{2} i F_{1212}^{X_{a b}}(\theta)=m_{a} m_{b} e^{(b-a) \theta} \partial_{\theta} S_{12}(\theta) .
$$

Expanding $\Psi_{i}$ by $X_{a b}$, and comparing (11) with (13) and (12) with (14), we arrive at the relation

$$
\begin{aligned}
\Psi_{i}= & -X_{11} \partial_{i} \ln m_{1}-X_{12} \partial_{i} \ln \left(m_{1} m_{2} e^{-\sigma}\right)^{1 / 2} \\
& -X_{22} \partial_{i} \ln m_{2}-X_{21} \partial_{i} \ln \left(m_{1} m_{2} e^{\sigma}\right)^{1 / 2} .
\end{aligned}
$$

A similar relation for $\bar{\Psi}_{i}$ is obtained by replacing $\partial_{i}$ with $\bar{\partial}_{i}$. The consistency of $\left\langle\Psi_{i}\right\rangle$ from (10) and (15) gives $\left\langle X_{12}\right\rangle=$ $\frac{1}{2} m_{1} m_{2} e^{-\sigma}$ and $\left\langle X_{21}\right\rangle=\frac{1}{2} m_{1} m_{2} e^{\sigma}$. Together with these results, we restrict the mass-coupling relation from conservation laws in the following.

UV conserved charges. - In the UV CFT, any element of the chiral algebra, $\Lambda(z)$, is a component of a conserved current: $\bar{\partial} \Lambda(z)=0$. Once we switch on the perturbation, this is no longer true, but we can systematically calculate the corrections. The leading order formula is

$$
\bar{\partial} \Lambda(z, \bar{z})=-\lambda_{i} \bar{\lambda}_{j} \oint_{z} \frac{d w}{2 i} \Lambda(z) \Phi_{i j}(w, \bar{z}) .
$$

Comparing the dimensions on the two sides, one can show that higher order terms cannot contribute, and the first order formula is actually exact.

Given (16), conserved currents are found by the counting argument [30,31]. For example, at the second level, we have three operators: the Ising stress tensor $L^{(1)}(z)$, the TCI one $L^{(2)}(z)$, and the product $L^{(3)}(z)=\psi(z) G(z)$. By analyzing carefully their operator product expansion (OPE) with the perturbing fields, $\Phi_{i j}$, we find two conservation laws. The first combination is the conservation of the energy $L=L^{(1)}+L^{(2)}$,

$$
\bar{\partial} L=\pi(1-h) \lambda_{i} \partial \Psi_{i},
$$

where $h=\frac{3}{5}$ is the chiral conformal dimension of the perturbing operators. The conservation of the other combination,

$$
J^{-}=L^{(1)}+\alpha L^{(3)}, \quad \alpha=\frac{\sqrt{5}}{4} \frac{\lambda_{1}}{\lambda_{2}},
$$

follows from the singular part of the $\mathrm{OPE} J^{-}(z) \lambda_{i} \Phi_{i j}(w, \bar{w})=$ $\frac{3}{2}\left[v_{i} \Phi_{i j}(w, \bar{w}) /(z-w)^{2}\right]+\frac{5}{2}\left[v_{i} \partial \Phi_{i j}(w, \bar{w}) /(z-w)\right]$ as

$$
\bar{\partial} J^{-}=\partial J^{+} \equiv v_{i} \partial \Psi_{i}
$$

where $v_{1}=(\pi / 2) \lambda_{1}$ and $v_{2}=(\pi / 6)\left(\lambda_{1}^{2} / \lambda_{2}\right)$. We denote the corresponding conserved charge by $Q$. Clearly, we have similar equations for the antichiral half, $\bar{J}^{-}$and $\bar{J}^{+}$. We can also calculate how the charge $Q$ acts on $\bar{J}^{-}$: $\left[Q, \bar{J}^{-}(z, \bar{z})\right]=-\pi \oint(d w / 2 \pi i) J^{-}(w) \bar{v}_{j} \bar{\Psi}_{j}(z, \bar{z})$. Using the short distance OPEs, we obtain

$$
\left[Q, \bar{J}^{-}\right]=-\frac{5}{2} v_{i} \bar{v}_{j} \partial \Phi_{i j}
$$

IR conserved charges.-From the two conservation laws for $L$ and for $J^{-}$, it is clear that they have linear combinations $\tau_{i}$ such that $\Psi_{i}$ satisfies $\partial \Psi_{i}=\bar{\partial} \tau_{i}$ for $i=1,2$, and similarly, for $\bar{\Psi}_{i}$. As a consequence, $F^{\Psi_{i}} \propto P^{+}$and $F^{\bar{\Psi}_{i}} \propto P^{-}$, which together with (8) and (15) give the relations

$$
\partial_{i} \ln \left(\frac{m_{1}}{m_{2}} e^{-\sigma}\right)=0, \quad \bar{\partial}_{i} \ln \left(\frac{m_{1}}{m_{2}} e^{\sigma}\right)=0 .
$$

Now it is advantageous to introduce the parameters

$$
\mu_{a}=\frac{m_{a}}{2} e^{\sigma_{a}}, \quad \bar{\mu}_{a}=\frac{m_{a}}{2} e^{-\sigma_{a}} .
$$

All physical combinations depend only on the difference of $\sigma_{a}$, namely, $\sigma=\sigma_{1}-\sigma_{2}$. The equations above imply that $\mu_{1} / \mu_{2}$ depends only on $\eta=\lambda_{1} / \lambda_{2}$ and $\bar{\mu}_{1} / \bar{\mu}_{2}$ on $\bar{\eta}=\bar{\lambda}_{1} / \bar{\lambda}_{2}$. In this notation, $\left\langle X_{12}\right\rangle=2 \mu_{2} \bar{\mu}_{1}$ and $\left\langle X_{21}\right\rangle=2 \mu_{1} \bar{\mu}_{2}$.

The action of the conserved currents and charges on multiparticle states are found using their forms such as (15), (19) with (21), and the relevant form factors given above. The commutator $\left[Q, \bar{J}^{-}\right]$is thus expressed in terms of the IR basis $X_{a b}$. Comparing the resulting expression to the UV result (20), we can derive the relation

$$
\Phi_{i j}=-\frac{4}{5}\left(\partial_{i} \ln m_{a}\right)\left(\bar{\partial}_{j} \ln m_{b}\right) X_{b a} .
$$

Master formula.-Our final ingredient for the masscoupling relation is the master formula, which is a generalization of the $\Theta$ sum rule of [32] for a conserved spin two current. Let us assume that $Y^{\mu \nu}$ satisfies $\partial_{\mu} Y^{\mu \nu}=0$ and that $\Psi$ is some scalar operator, such that the leading term of their conformal OPE is 
$\left\langle Y^{--}(z) \Psi(0)\right\rangle=C(0) / z^{2}+\cdots$. By following the calculation that leads to the $\Theta$ sum rule, we obtain

$$
\int d^{2} x\left\langle Y^{+-}(x) \Psi(0)\right\rangle_{c}=-\pi C(0),
$$

where $\langle\cdot\rangle_{c}$ stands for the connected part. For this, we used relativistic invariance to parametrize the two point function as $\left\langle Y^{\mu \nu}(x) \Psi(0)\right\rangle_{c}=-x^{\mu} x^{\nu} r^{-4} C\left(r^{2}\right)+\eta^{\mu \nu} A\left(r^{2}\right)+\epsilon^{\mu \nu} B\left(r^{2}\right)$. The conservation law then leads to $G / r^{2}=$ $\left(d / d r^{2}\right)(C+G)$, where $G=C+2 A+2 B$. In massive theories $C(\infty)=G(\infty)=0$ and a relevant conformal dimension, $\Delta<1$, for $\Psi$ implies $G(0)=0$.

Applying these formulas to the stress tensor, we recover the $\Theta$ sum rule: $\int d^{2} x\langle\Theta(x) \Psi(0)\rangle_{c}=-2 \Delta\langle\Psi\rangle$. Since the second tensor index of $Y^{\mu \nu}$ can be regarded as a label of the current, the formula can be applied to the other conserved current $J^{\mu} \sim Y^{\mu-}$. This leads to a differential equation for the mass-coupling relation.

Mass-coupling relation.-To see this, first note that the master formula (24) enables us to calculate the free energy Ward identity,

$\partial_{i} \bar{\partial}_{j} \mathcal{F}=-\left\langle\Phi_{i j}\right\rangle-\int d^{2} x\left\langle\Psi_{i}(x) \bar{\Psi}_{j}(0)\right\rangle_{c}=-\frac{5}{2}\left\langle\Phi_{i j}\right\rangle$.

Together with $\mathcal{F}=\mu_{1} \bar{\mu}_{2}+\mu_{2} \bar{\mu}_{1}$, this implies complete factorization; i.e., $\mu_{a}$ depends on $\lambda_{i}$ as $\mu_{a}\left(\lambda_{1}, \lambda_{2}\right)$, and similarly, $\bar{\mu}_{a}$ as $\bar{\mu}_{a}\left(\bar{\lambda}_{1}, \bar{\lambda}_{2}\right)$. This means that the original three-variable mass-coupling relation is reduced to two identical copies of the chiral two-variable mass-coupling relation. On dimensional grounds, we can thus write

$$
\mu_{a}=\frac{\lambda_{1}^{5 / 2}}{2} q_{a}(\eta), \quad \bar{\mu}_{a}=\frac{\bar{\lambda}_{1}^{5 / 2}}{2} q_{a}(\bar{\eta}),
$$

so as to maintain the left-right symmetry of the problem, where as before $\eta=\lambda_{1} / \lambda_{2}$.

The master formula implies also that

$$
v_{i} \partial_{i}\left\langle\Phi_{k j}\right\rangle=\int d^{2} x\left\langle J^{+}(x) \Phi_{k j}\right\rangle_{c}=\frac{\pi}{2} M_{k i}\left\langle\Phi_{i j}\right\rangle,
$$

where from the OPEs, we obtain $M_{11}=1$, $M_{12}=M_{21}=\frac{1}{2} \eta$, and $M_{22}=0$. Through (23), this actually translates into the following differential equation for $q_{a}$ :

$$
\eta^{2}\left(1-\frac{\eta^{2}}{3}\right) q_{a}^{\prime \prime}+\eta\left(4-\frac{2 \eta^{2}}{3}\right) q_{a}^{\prime}+\frac{5}{4} q_{a}=0,
$$

which is a hypergeometric differential equation whose solutions need to be fixed from the boundary conditions. One special case can be obtained by sending $\lambda_{1}=\bar{\lambda}_{1}$ to 0 . In this case, only the TCI model is perturbed with $\lambda_{2} \bar{\lambda}_{2} \Phi_{22}$, and the masses are explicitly known as $m_{1}=0$ and $m_{2}=\kappa\left(\lambda_{2} \bar{\lambda}_{2}\right)^{5 / 4}$ with $\kappa=\left[56(21 \pi)^{1 / 4} / 5^{5 / 2}\right]\left\{\left[\Gamma\left(-\frac{7}{5}\right) \Gamma\left(\frac{1}{5}\right)\right] /\right.$ $\left.\left[\Gamma\left(\frac{12}{5}\right) \Gamma\left(\frac{4}{5}\right)\right]\right\}[13,22]$. The solution of (28) for such vanishing $\mu_{1}$ is unique up to normalization, giving
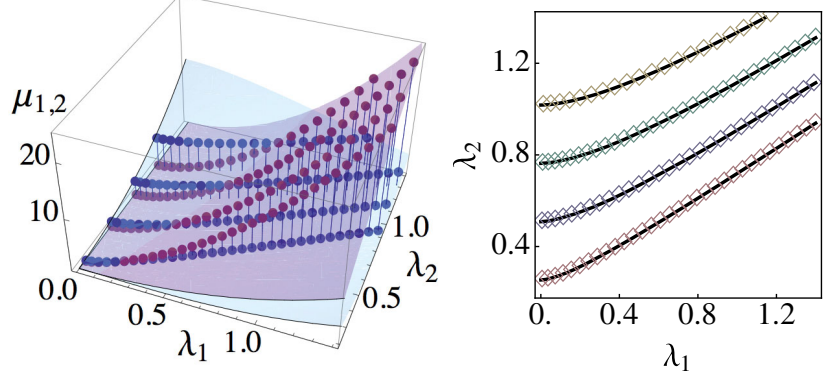

FIG. 1. Plots of $\left(\mu_{1}, \mu_{2}\right)$ versus $\left(\lambda_{1}, \lambda_{2}\right)$. On the left, the red and blue surfaces represent $\mu_{1}\left(\lambda_{i}\right)$ and $\mu_{2}\left(\lambda_{i}\right)$ in (29) and (30), respectively. The red and blue points represent the numerical data $\left[\lambda_{1}\left(\mu_{a}\right), \lambda_{2}\left(\mu_{a}\right), \mu_{b}\right](b=1,2)$ from the TBA equations, which are solved for given $\mu_{a}=\bar{\mu}_{a}$. Each sequence from the bottom to the top corresponds to $\left(\mu_{2}\right)^{2 / 5}=1 / 2,1,3 / 2,2$, with $\mu_{1}$ varied. $\lambda_{i}$ are determined by comparing the TBA free energy with the CFT perturbation. On the right, the diamonds $(\diamond)$ represent the projections of the left points to the $\left(\lambda_{1}, \lambda_{2}\right)$ plane. The solid lines are the contours in the fundamental domain for $\left[\mu_{2}\left(\lambda_{i}\right)\right]^{2 / 5}=1 / 2,1,3 / 2,2$ from (30).

$$
\mu_{1}\left(\lambda_{1}, \lambda_{2}\right)=B \lambda_{1}^{2}\left(\lambda_{1}+\sqrt{3} \lambda_{2}\right)^{1 / 2} F\left(\frac{2 \lambda_{1}}{\lambda_{1}+\sqrt{3} \lambda_{2}}\right),
$$

where $F(z)={ }_{2} F_{1}\left(-\frac{1}{2}, \frac{3}{2} ; 3 \mid z\right)$. The $S_{3}$ symmetry then yields

$$
\mu_{2}\left(\lambda_{1}, \lambda_{2}\right)=\frac{B}{4} \frac{\left(\sqrt{3} \lambda_{2}-\lambda_{1}\right)^{2}}{\left(\lambda_{1}+\sqrt{3} \lambda_{2}\right)^{-1 / 2}} F\left(\frac{\sqrt{3} \lambda_{2}-\lambda_{1}}{\lambda_{1}+\sqrt{3} \lambda_{2}}\right) .
$$

(29) and (30) hold in the fundamental domain $0 \leq \lambda_{1} \leq \sqrt{3} \lambda_{2}$, which are continued outside by the $S_{3}$ symmetry. The normalization is fixed by the above single-mass result: $B=\kappa(5 \pi / 16 \sqrt[4]{3})$. This is our main result, which we have checked numerically from the TBA equations [19]. Figure 1 shows the agreement of (29), (30), and samples of numerical data. Furthermore, at $\left(\lambda_{1}, \lambda_{2}\right)=(\lambda / 2, \sqrt{3} \lambda / 2)$, we confirm that $\mu_{1}=\mu_{2}=$ $(B / 2 \sqrt{2}) F(1 / 2) \lambda^{5 / 2}$, which exactly reproduces the masscoupling relation in the equal-mass case [14,22]. The mass-coupling relation enables us to express the free energy density $\mathcal{F}$ in terms of $\left(\lambda_{i}, \bar{\lambda}_{i}\right)$, which then can be used via (25) to obtain the one-point functions of $\Phi_{i j}$.

Conclusions.-In this Letter, we developed a new method to calculate the exact mass-coupling relation for multiscale quantum integrable models. We combined form factor perturbation theory with the construction of conserved tensor currents. The generalization of the $\Theta$ sum rule Ward identity of these currents provided a differential equation for the mass-coupling relations, leading to solutions in terms of hypergeometric functions. This is the first result for multiscale mass-coupling relations. Our work provides the missing link to develop an analytic expansion of ten-particle scattering amplitudes of the fourdimensional maximally supersymmetric gauge theory at strong coupling around a $\mathbb{Z}_{10}$-symmetric kinematic point 
[33]. Although we analyzed here the simplest multiscale HSG model, the methods can be extended for other multiscale perturbed CFTs. More details and related results will be reported elsewhere [34].

We would like to thank J. Luis Miramontes for useful conversations. This work was supported by Japan-Hungary Research Cooperative Program. Z. B., J. B., and G. Zs. T. were supported by a Lendület Grant and by OTKA Grant No. K116505, whereas K. I. and Y.S. were supported by JSPS Grant-in-Aid for Scientific Research, Grant No. 15K05043 and No. 24540248.

*bajnok.zoltan@wigner.mta.hu

†balog.janos@wigner.mta.hu

*ito@th.phys.titech.ac.jp

§satoh@het.ph.tsukuba.ac.jp

toth.gabor.zsolt@wigner.mta.hu

[1] P. Hasenfratz, M. Maggiore, and F. Niedermayer, The exact mass gap of the $\mathrm{O}(3)$ and $\mathrm{O}(4)$ nonlinear $\sigma$-models in $d=2$, Phys. Lett. B 245, 522 (1990).

[2] P. Hasenfratz and F. Niedermayer, The exact mass gap of the $\mathrm{O}$ (N) $\sigma$-model for arbitrary $N \geq 3$ in $d=2$, Phys. Lett. B 245, 529 (1990).

[3] A. B. Zamolodchikov, Thermodynamic Bethe ansatz in relativistic models. Scaling three state Potts and Lee-Yang models, Nucl. Phys. B342, 695 (1990).

[4] P. Forgács, F. Niedermayer, and P. Weisz, The exact mass gap of the Gross-Neveu model (I). The thermodynamic Bethe ansatz, Nucl. Phys. B367, 123 (1991).

[5] P. Forgács, S. Naik, and F. Niedermayer, The exact mass gap of the chiral Gross-Neveu model, Phys. Lett. B 283, 282 (1992).

[6] J. Balog, S. Naik, F. Niedermayer, and P. Weisz, Exact mass gap of the chiral $\mathrm{SU}(n) \times \mathrm{SU}(n)$ model, Phys. Rev. Lett. 69, 873 (1992).

[7] V. A. Fateev, E. Onofri, and A. B. Zamolodchikov, Integrable deformations of $\mathrm{O}(3)$ sigma model. The sausage model, Nucl. Phys. B406, 521 (1993).

[8] T. J. Hollowood, The exact mass gaps of the principal chiral models, Phys. Lett. B 329, 450 (1994).

[9] J. M. Evans and T. J. Hollowood, The exact mass gap of the supersymmetric $\mathrm{O}(N)$ sigma model, Phys. Lett. B 343, 189 (1995).

[10] J. M. Evans and T. J. Hollowood, The exact mass gap of the supersymmetric $\mathrm{CP}^{n-1}$ sigma model, Phys. Lett. B 343, 198 (1995).

[11] A. B. Zamolodchikov, Two point correlation function in scaling Lee-Yang model, Nucl. Phys. B348, 619 (1991).

[12] F. Constantinescu and R. Flume, The convergence of strongly relevant perturbations of $d=2$ conformal field theories, Phys. Lett. B 326, 101 (1994).

[13] A. B. Zamolodchikov, Mass scale in the sine-Gordon model and its reductions, Int. J. Mod. Phys. A 10, 1125 (1995).

[14] V.A. Fateev, The exact relations between the coupling constants and the masses of particles for the integrable perturbed conformal field theories, Phys. Lett. B 324, 45 (1994).
[15] C. R. Fernández-Pousa, M. V. Gallas, T. J. Hollowood, and J. L. Miramontes, The symmetric space and homogeneous sine-Gordon theories, Nucl. Phys. B484, 609 (1997).

[16] C. R. Fernández-Pousa, M. V. Gallas, T. J. Hollowood, and J. L. Miramontes, Solitonic integrable perturbations of parafermionic theories, Nucl. Phys. B499, 673 (1997).

[17] C. R. Fernández-Pousa and J. L. Miramontes, Semiclassical spectrum of the homogeneous sine-Gordon theories, Nucl. Phys. B518, 745 (1998).

[18] J. L. Miramontes and C. R. Fernández-Pousa, Integrable quantum field theories with unstable particles, Phys. Lett. B 472, 392 (2000).

[19] O. A. Castro-Alvaredo, A. Fring, C. Korff, and J. L. Miramontes, Thermodynamic Bethe ansatz of the homogeneous sine-Gordon models, Nucl. Phys. B575, 535 (2000).

[20] P. Dorey and J. L. Miramontes, Mass scales and crossover phenomena in the homogeneous sine-Gordon models, Nucl. Phys. B697, 405 (2004).

[21] Y. Hatsuda, K. Ito, K. Sakai, and Y. Satoh, Thermodynamic Bethe ansatz equations for minimal surfaces in $\mathrm{AdS}_{3}$, J. High Energy Phys. 04 (2010) 108.

[22] Y. Hatsuda, K. Ito, K. Sakai, and Y. Satoh, g-functions and gluon scattering amplitudes at strong coupling, J. High Energy Phys. 04 (2011) 100.

[23] Y. Hatsuda, K. Ito, and Y. Satoh, T-functions and multigluon scattering amplitudes, J. High Energy Phys. 02 (2012) 003.

[24] Y. Hatsuda, K. Ito, and Y. Satoh, Null-polygonal minimal surfaces in $\mathrm{AdS}_{4}$ from perturbed $\mathrm{W}$ minimal models, J. High Energy Phys. 02 (2013) 067.

[25] L. F. Alday and J. M. Maldacena, Gluon scattering amplitudes at strong coupling, J. High Energy Phys. 06 (2007) 064.

[26] Č. Crnković, R. Paunov, G. M. Sotkov, and M. Stanishkov, Fusions of conformal models, Nucl. Phys. B336, 637 (1990).

[27] O. A. Castro-Alvaredo, A. Fring, and C. Korff, Formfactors of the homogeneous sine-Gordon models, Phys. Lett. B 484, 167 (2000).

[28] O. A. Castro-Alvaredo and A. Fring, Identifying the operator content, the homogeneous sine-Gordon models, Nucl. Phys. B604, 367 (2001).

[29] G. Delfino, G. Mussardo, and P. Simonetti, Nonintegrable quantum field theories as perturbations of certain integrable models, Nucl. Phys. B473, 469 (1996).

[30] A. B. Zamolodchikov, Integrals of motion in scaling 6 three state Potts model field theory, Int. J. Mod. Phys. A 03, 743 (1988).

[31] A. B. Zamolodchikov, Integrable field theory from conformal field theory, Adv. Stud. Pure Math. 19, 641 (1989).

[32] G. Delfino, P. Simonetti, and J. L. Cardy, Asymptotic factorization of form-factors in two-dimensional quantum field theory, Phys. Lett. B 387, 327 (1996).

[33] In [22], the mass-coupling relation was studied assuming that $\lambda_{i}$ are polynomials of $\mu_{a}^{2 / 5}$. In the second order CFT perturbation of $\mathcal{F}$, that leads to a deviation of less than $1 \%$ from the exact values for each contribution from the chiral and antichiral sectors. It is still unclear why such a simple assumption works well effectively.

[34] Z. Bajnok, J. Balog, K. Ito, Y. Satoh, and G. Z. Tóth, On the mass-coupling relation of multi-scale quantum integrable models, arXiv:1604.02811. 